\title{
Three Types of Nuclear Envelope Assemblies Associated with Micronuclei
}

\author{
Kohei Miyazaki'1, Yuichi Ichikawa², Noriko Saitoh ${ }^{2}$, Hisato Saitoh ${ }^{1,3 *}$ \\ ${ }^{1}$ Department of Biological Science, Graduate School of Science and Technology, Kumamoto University, Kumamoto, Japan \\ ${ }^{2}$ Division of Cancer Biology, The Cancer Institute of Japanese Foundation for Cancer Research (JFCR), Ariake, Koto-ku, Japan \\ ${ }^{3}$ Faculty of Advanced Science and Technology (FAST), Kumamoto University, Kumamoto, Japan \\ Email: *hisa@kumamoto-u.ac.jp
}

How to cite this paper: Miyazaki, K. Ichikawa, Y., Saitoh, N. and Saitoh, H. (2020) Three Types of Nuclear Envelope Assemblies Associated with Micronuclei. CellBio, 9, 14-28.

https://doi.org/10.4236/cellbio.2020.91002

Received: December 20, 2019

Accepted: January 11, 2020

Published: January 14, 2020

Copyright (c) 2020 by author(s) and Scientific Research Publishing Inc. This work is licensed under the Creative Commons Attribution International License (CC BY 4.0).

http://creativecommons.org/licenses/by/4.0/

\section{Open Access}

\begin{abstract}
While micronuclei (MN) store extranuclear DNA and cause genome instability, the effects of nuclear envelope (NE) assembly defects associated with $\mathrm{MN}$ on genome instability remain largely unknown. Here, we investigated the NE protein distribution in MN using HeLa human cervical cancer cells. Under the standard condition and two pharmacological culture conditions, we found that three types of NE protein assemblies were associated with $\mathrm{MN}$ : 1) intact NE assembly, in which both core and non-core NE proteins were evenly present; 2) type I assembly, in which only core NE proteins were detectable; and 3) type II assembly in which a region deficient for both core and non-core NE proteins existed and a pattern recognition receptor, cyclic guanosine monophosphate-adenosine monophosphate synthase, was frequently detected. Our findings provide experimental settings and a method of grouping $\mathrm{MN}$-associated NE defects, which may be helpful for researchers who are interested in regulation of genome and nuclear organization relevant to cancer development.
\end{abstract}

\section{Keywords}

Cancer, Lamin, Micronucleus, Nuclear Envelope, Paclitaxel/Taxol

\section{Introduction}

In nearly all eukaryotic cells, genomic DNA is enclosed in the nucleus and the boundary of the nucleus is the nuclear envelope (NE) [1]. NEs are composed of a double membrane sheet of two closely juxtaposed lipid bilayers referred to as the inner and outer nuclear membranes. Along the inner and/or outer membranes, many different kinds of proteins interact with and/or are embedded in them. Perturbation of NE assembly because of mutations in NE proteins is asso- 
ciated with cell senescence and certain diseases, most notably cancer, indicating the importance of regulated NE assembly for proper cellular activities [1]. Although the precise regulation of $\mathrm{NE}$ assembly remains largely unknown, $\mathrm{NE}$ proteins can be categorized into two groups, "core" and "non-core", based on the patterns of NE protein association with respect to the domains that are transiently formed around decondensing chromosomes during telophase of mitosis [2] [3] [4].

Micronuclei (MN) are small nuclei that store extranuclear chromatin bodies in cells. A major cause of $\mathrm{MN}$ formation is miss-segregation of chromosomal DNA during mitosis, followed by assembly of NE proteins around the miss-segregated chromosomal DNA in the extranuclear space of the cytoplasm [5] [6] [7] [8]. Recently, Liu et al. reported that the MN boundary frequently contains insufficiently assembled NE proteins. Specifically, core NE proteins can be assembled more efficiently around miss-segregated chromosomes than non-core NE proteins, leading to NE protein assembly defects in $\mathrm{MN}$ [8]. Although their findings concerning $\mathrm{NE}$ assemblies associated with $\mathrm{MN}$ provide a new perspective to assess the linkage between $\mathrm{NE}$ assembly defects and cell fate decisions of MN-containing cells, they may have overlooked other types of NE assembly defects that might regulate such decisions.

In this study, we investigated the localization of NE proteins in HeLa human cervical cancer cells. Based on the classification of core- and non-core NE proteins, we found three types of NE protein assemblies associated with MN under the standard condition and two pharmacological cell culture conditions. Interestingly, one of the NE types was characterized by a region deficient for both core and non-core NE proteins, and $\mathrm{MN}$ with this type of $\mathrm{NE}$ assembly frequently accumulated cyclic guanosine monophosphate-adenosine monophosphate synthase (cGAS), a pattern recognition receptor implicated in non-self DNA recognition. Our findings describe pharmacological cell culture conditions to induce $\mathrm{MN}$ and a method to differentiate $\mathrm{NE}$ defects associated with $\mathrm{MN}$, providing insights into quality control of $\mathrm{MN}$ and cell fate decisions of $\mathrm{MN}$-containing cells, which are relevant to regulation of genome instability and cancer development.

\section{Materials and Methods}

\subsection{Reagents and Antibodies}

All reagents, antibodies, and stock solutions are listed in the Supplementary information.

\subsection{Culture Conditions and Drug Treatments}

HeLa cells were maintained in Dulbecco's modified Eagle's medium/Ham's F-12 nutrient mixture containing $5 \%$ fetal calf serum and antibiotics at $37^{\circ} \mathrm{C}$ in a $5 \%$ $\mathrm{CO}_{2}$ incubator. For pharmacological induction of $\mathrm{MN}$, we used the following two conditions. Low paclitaxel conditions: $5 \times 10^{5}$ cells on coverslips in 12-well plates were cultured in the presence of $10 \mathrm{nM}$ paclitaxel for $18 \mathrm{~h}$ [9] [10] [11]. 
High paclitaxel and reversine conditions: $5 \times 10^{5}$ cells on coverslips in 12-well plates were cultured in the presence of $1 \mu \mathrm{M}$ paclitaxel for $16 \mathrm{~h}$, followed by incubation for $2 \mathrm{~h}$ with $200 \mathrm{ng} / \mathrm{ml}$ reversine [12]. Time-lapse analyses were performed to visualize $\mathrm{MN}$ formation under the pharmacological conditions (see Supplementary procedure).

\subsection{Indirect Immunofluorescence Analysis and Image Processing}

Indirect immunofluorescence analysis of cells cultured on coverslips was performed as follows. Cells were fixed with $4 \%$ paraformaldehyde in phosphate buffered saline (PBS) for $10 \mathrm{~min}$, followed by treatment with $0.1 \%$ Triton X-100 in PBS for $5 \mathrm{~min}$. After blocking with $1 \%$ bovine serum albumin in PBS for $15 \mathrm{~min}$ at room temperature $\left(17^{\circ} \mathrm{C}-20^{\circ} \mathrm{C}\right)$, the cells were labeled with primary and secondary antibodies accordingly. During secondary antibody incubation, samples were costained with Hoechst 33342 to visualize DNA. All steps were carried out at room temperature. Samples were observed using the XI71 microscope imaging system and cellSens standard software (Olympus). For each image, at least three slices from the z-axis were captured. ImageJ was used for image processing.

\section{Results and Discussion}

\subsection{NE Protein Assembly Defects in Asynchronously Cultured HeLa Cells}

Liu et al. reported that core NE proteins assemble efficiently around lagging chromosomes to form $\mathrm{MN}$, whereas non-core NE proteins assemble poorly [8]. To reproduce their observation, we performed immunostaining analyses of $\mathrm{MN}$ in exponentially growing HeLa cells. We used antibodies against lamin A/C and emerin to detect core NE proteins and antibodies against lamin B and Ran-binding protein 2/nucleoporin 358 (RanBP2/Nup358) to detect non-core NE proteins (see Table S1). As shown in Figure 1(a) and Figure S1(a), we observed multiple $\mathrm{MN}$ enriched with lamin $\mathrm{A} / \mathrm{C}$ and emerin (core) but deficient for lamin $\mathrm{B}$ and RanBP2 (non-core), reproducing Liu et al.'s observation. However, we also noticed significant numbers of $\mathrm{MN}$ with seemingly different $\mathrm{NE}$ protein distributions. Specifically, we observed MN with NE regions deficient for both core and non-core NE proteins (Figure 1(b) and Figure S1(b)). Therefore, we observed three types of NE protein assemblies associated with MN under our experimental settings: 1) NEs enriched with core NE proteins, but deficient for non-core NE proteins referred to as type I NE protein assembly; 2) NEs containing regions deficient for both core and non-core NE proteins designated as type II NE protein assembly; and 3) no detectable NE protein deficiency called intact NE assembly (Figure 1(c) and Figure S1(c)). It should be noted that we did not observe any MN exhibiting NE regions enriched with non-core NE proteins, but deficient for core NE proteins $(\sim 0 \%)$. The percentages of $\mathrm{MN}$ with type I and 

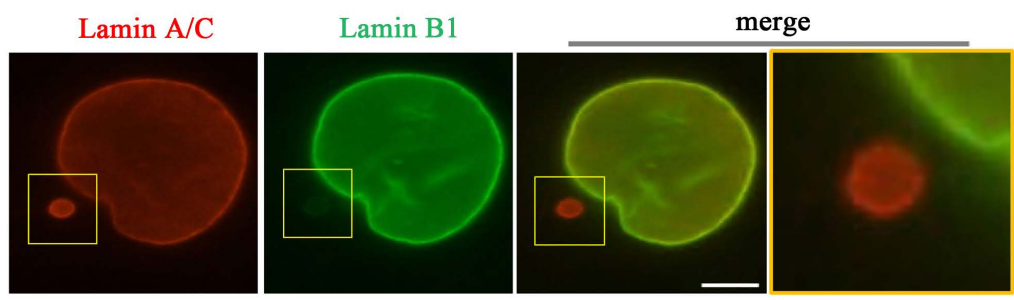

(a)
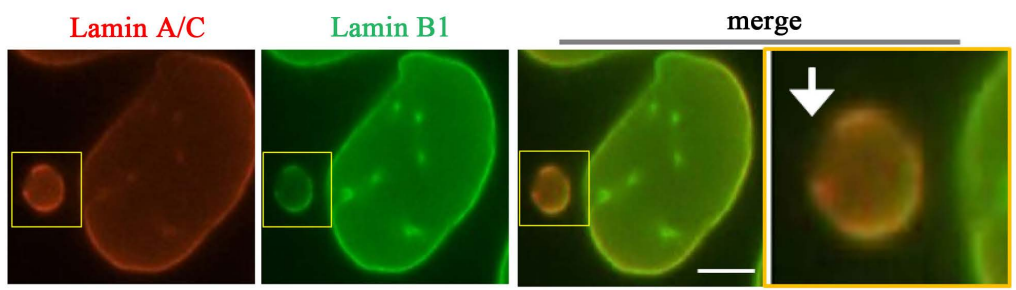

(b)
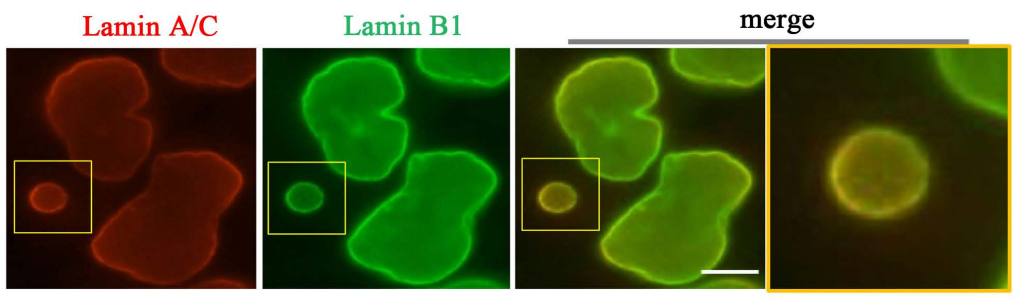

(c)

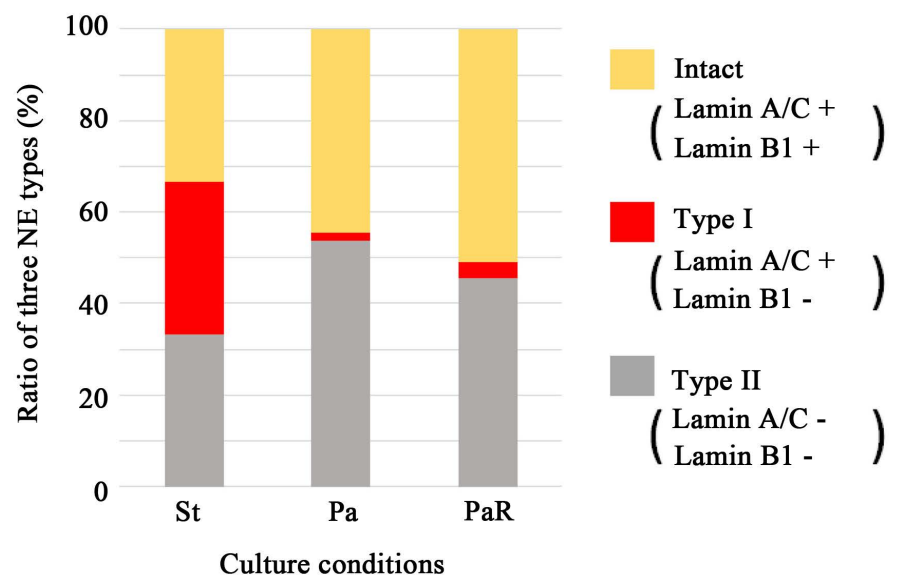

(d)

Figure 1. Association of three types of NE assembly with $\mathrm{MN}$ in asynchronously cultured HeLa cells. (a)-(c) Exponentially growing HeLa cells subjected to immunostaining analysis using anti-lamin A/C (left) and anti-lamin B1 (middle left) antibodies. Merged images are shown in the middle right. The area in the yellow box is enlarged to the right. The arrow in B represents the region lacking both lamin $\mathrm{A} / \mathrm{C}$ and lamin $\mathrm{B} 1$ signals. Scale bar: 20 $\mu \mathrm{m}$; (d) MN immunostaining patterns were categorized into three types: MN with seemingly no defects in lamin $\mathrm{A} / \mathrm{C}$ and lamin B1 assemblies, designated as "intact" (positive for lamin $\mathrm{A} / \mathrm{C}$ and lamin B1; yellow box), $\mathrm{MN}$ enriched with lamin $\mathrm{A} / \mathrm{C}$ but deficient for lamin B1, designated as "type I" (red box), and MN containing a region deficient for both lamin $\mathrm{A} / \mathrm{C}$ and lamin $\mathrm{B} 1$ designated "type II" (gray box). Sixty MN were detected under the asynchronous culture conditions (St; left bar graph), low paclitaxel concentration conditions (Pa; middle bar graph), or paclitaxel plus reversine conditions (PaR; right bar graph). 
type II defects, and intact NE protein assembly were approximately $28 \%, 34 \%$, and 38\%, respectively, in 60 cells containing MN (left columns in Figure 1(d)).

\subsection{Induction of MN with Type II NE Protein Assembly under Two Pharmacological Conditions}

Because type II NE assembly appeared to represent a previously uncharacterized mode for storing DNA in $\mathrm{MN}$, we were interested in determining whether such NE protein assembly emerged in cells under culture conditions distinct from the standard asynchronous conditions ("St" in Figure 2(a)). For this purpose, we chose the following two pharmacological treatments. The first set of conditions exposed cells to paclitaxel at a low concentration, which did not promote mitotic arrest but produced dramatic segregation errors, leading to $\mathrm{MN}$ production ("Pa" in Figure 2(a) and Movie S1) [9] [10] [11]. The second set of conditions employed a high dose of paclitaxel, under which mitotic arrest was induced, followed by exposure to reversine that overrides mitosis by inhibiting mitotic kinase Aurora B, resulting in MN generation ("PaR" in Figure 2(a) and Movie S2) [12]. As shown in Figure 2(b), both pharmacological conditions efficiently induced MN; approximately 60 and $80 \%$ of the cells contained MN under the $\mathrm{Pa}$ or PaR conditions, respectively. But it should be mentioned that the numbers of $\mathrm{MN}$ in a cell were different under the two conditions; approximately $6 \mathrm{MN}$ and $24 \mathrm{MN}$ were present per cell under the $\mathrm{Pa}$ and $\mathrm{PaR}$ conditions, respectively (Figure 2(c)).

To detect type II NE assembly under the two pharmacological conditions, $\mathrm{Pa}$ and $\mathrm{PaR}$, we performed immunostaining analyses using antibodies against lamin $\mathrm{A} / \mathrm{C}$ and emerin to detect core NE proteins or lamin B and RanBP2 to detect non-core NE proteins. As shown in Figure 2(c), Figure 2(d), Figure S2(a) and Figure S2(b), we observed MN with type II NE protein assembly under both pharmacological conditions. The percentage of type II NE assembly was approximately 53\% under $\mathrm{Pa}$ conditions and $46 \%$ under $\mathrm{PaR}$ conditions (middle and right columns, respectively, in Figure $1(\mathrm{~d})$ ). It should be noted that there were some ( $5 \%)$ cells with type I NE assembly under both Pa and PaR conditions and we did not observe any $\mathrm{MN}$ exhibiting $\mathrm{NE}$ regions enriched with non-core NE proteins, but deficient for core NE proteins ( 0\%). These results suggested that, although the ratio of $\mathrm{NE}$ assembly types was somehow different from that obtained under standard conditions, three types of NE protein assembly were formed under all culture conditions and type II appeared to represent a major mode of NE protein assembly associated with MN.

\subsection{Uneven DNA Distribution in MN with the Type II NE Protein Assembly Defect}

Considering that interplay between NE proteins and DNA has a pivotal role in NE formation and chromatin architecture [1] [2] [3] [4], we were interested in the DNA distribution of MN with type II NE assembly. To assess the DNA distribution in $\mathrm{MN}$ with type II protein assembly as well as other types of NE 


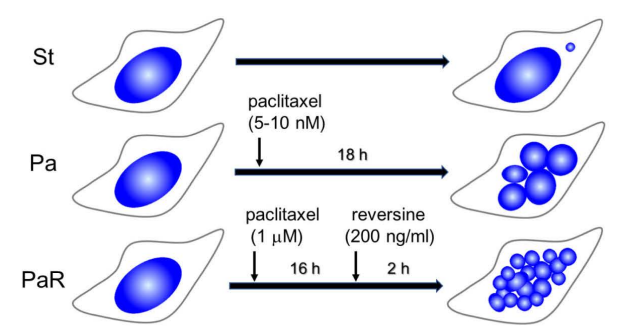

(a)

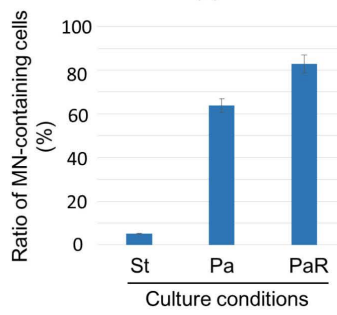

(b)

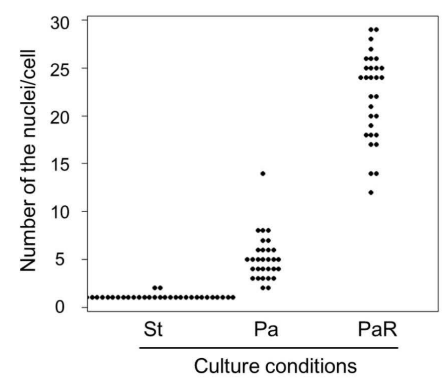

(c)

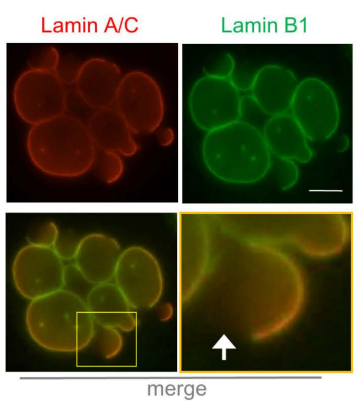

(d)

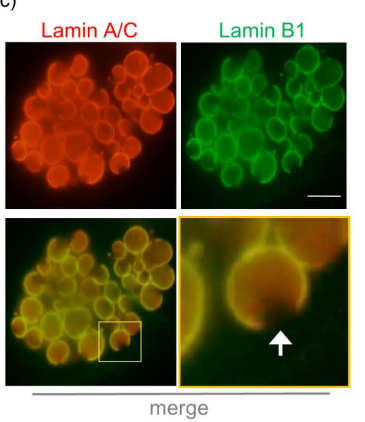

(e)

Figure 2. Generation of MN under the standard condition and two pharmacological culture conditions. (a) Schematic representations of the standard and two drug-treated procedures. The expected MN morphologies under each condition are illustrated on the right. St, standard conditions; $\mathrm{Pa}$, treatment with a low paclitaxel concentration; PaR, treatment with a high paclitaxel concentration followed by reversine exposure; (b) After the numbers of MN-containing cells under the St, $\mathrm{Pa}$ and $\mathrm{PaR}$ conditions were counted, the $\mathrm{MN}$-containing cell ratio of each condition is calculated and represented in a bar graph; (c) The number of nuclei in each cell under the St, Pa or PaR condition was counted in 30 cells and these numbers are represented in a point graph; (d) MN induced under Pa conditions were visualized by anti-lamin A/C (upper left) and anti-lamin B1 (upper left) antibodies. A merged image is shown at the lower left. The yellow box is enlarged at the lower right. The arrow represents the region lacking both lamin $\mathrm{A} / \mathrm{C}$ and lamin $\mathrm{B} 1$ signals. Scale bar: $20 \mu \mathrm{m}$; (e) MN induced under PaR conditions were visualized by anti-lamin $\mathrm{A} / \mathrm{C}$ (upper left) and anti-lamin B1 (upper right) antibodies. A merged image is shown at the lower left. The yellow box is enlarged at the lower right. The arrow represents the region lacking both lamin A/C and lamin B1 signals. Scale bar: $20 \mu \mathrm{m}$. 
assemblies, we used Hoechst 33342 for fluorescent DNA visualization in MN. Representative images are shown in Figures 3(a)-(c). While the intensity of Hoechst 33342 signals appeared to be evenly distributed in $\mathrm{MN}$ with intact and type I NE assembly, the signal intensity was unevenly distributed in MN with type II NE assembly. Specifically, the area proximal to the region deficient for
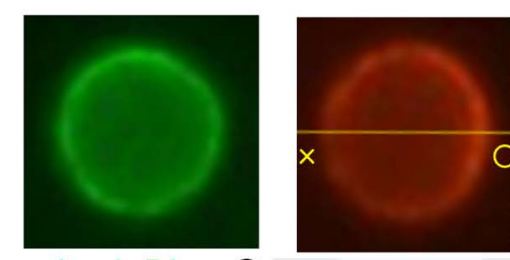

Lamin B1

Intact

$\left(\begin{array}{l}\operatorname{Lamin} A / C+ \\ \operatorname{Lamin} B 1+\end{array}\right)$
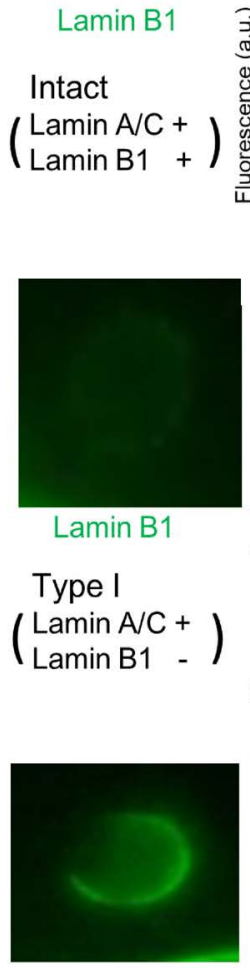

$\left(\begin{array}{l}\text { Lamin A/C - } \\ \text { Lamin B1 - }\end{array}\right)$

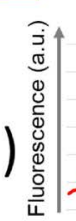

Lamin A/C

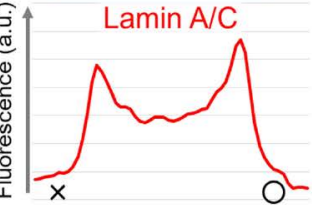

(a)
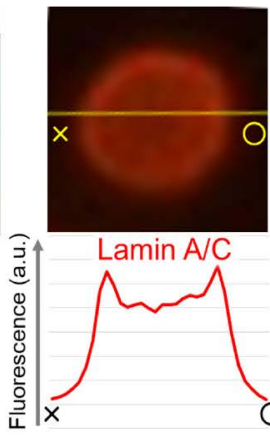

Lamin A

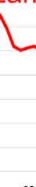

Lamin B1

Type I

$\left(\begin{array}{l}\operatorname{Lamin} A / C+ \\ \operatorname{Lamin} B 1-\end{array}\right)$

Lamin B1

Type II

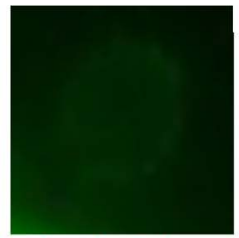

Lamin B1 -

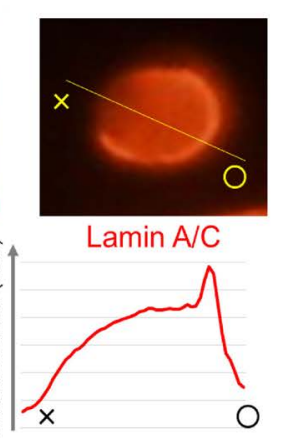

Lamin A/C

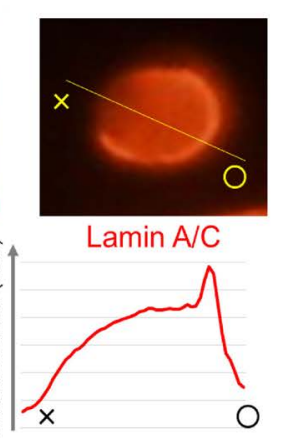

(c)

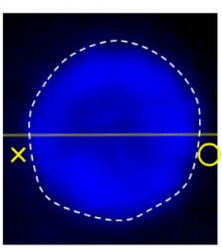

DNA

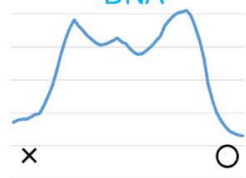

O
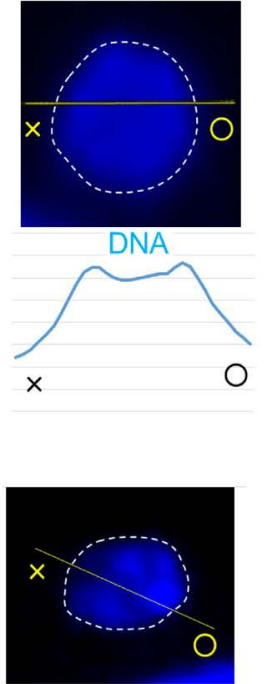

DNA

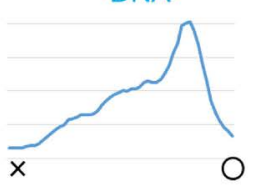

Figure 3. DNA distribution in MN with intact, type I, and type II NE assemblies. (a)-(c) Exponentially growing HeLa cells were subjected to indirect immunofluorescence analysis using anti-lamin B1 (upper left) and anti-lamin A/C (upper middle) antibodies. In (a), a representative image of $\mathrm{MN}$ with seemingly no defects in lamin $\mathrm{A} / \mathrm{C}$ and lamin $\mathrm{B} 1$ assemblies ("Intact NE") is shown. In (b), a representative image of MN enriched with lamin $\mathrm{A} / \mathrm{C}$ but deficient for lamin B1 ("Type I NE") is presented. In (c), a representative image of $\mathrm{MN}$ containing a region deficient for both lamin A/C and lamin B1 ("Type II NE”) is shown. The DNA distribution was visualized by Hoechst 33342 staining (upper right). Intensities of lamin $\mathrm{A} / \mathrm{C}$ and Hoechst 33342 staining were measured along the indicated lines $(x \rightarrow O)$ and are represented in line graphs accordingly (lower panels). Signal intensities are represented as arbitrary units (a. u.). 
both lamin $\mathrm{A} / \mathrm{C}$ core and lamin B1 non-core NE proteins exhibited less intense Hoechst 33342 signals, suggesting the existence of a DNA-poor area proximal to the region deficient for core and non-core NE proteins in $\mathrm{MN}$ with type II NE protein assembly. A similar uneven DNA distribution was detected in MN with type II NE protein assembly under the two pharmacological $\mathrm{Pa}$ and $\mathrm{PaR}$ culture conditions (Figure S3).

\section{4. cGAS Accumulation in MN with Type II NE Protein Assembly}

To further differentiate $\mathrm{MN}$ features with respect to NE types, we investigated accumulation of cGAS in MN under standard, $\mathrm{Pa}$, and $\mathrm{PaR}$ conditions. It is increasingly clear that cGAS, which exhibits higher affinity for naked DNA than DNA residing in the nucleosome/chromatin [13], has emerged as a pattern recognition receptor implicated in sensing self- versus non-self DNA, followed by activation of the STING (stimulator of interferon genes) pathway [14] [15] [16] and references therein]. We therefore expected that cGAS accumulation might be a suitable signal to evaluate the quality of DNA residing in MN with different types of NE protein assembly. When we performed immunostaining analyses using an anti-cGAS antibody, cGAS signals were detected more frequently in $\mathrm{MN}$ with the region deficient for lamin A/C indicative of type II NE assembly than in $\mathrm{MN}$ enriched with lamin A/C indicative of type I or intact NE assembly (Figure 4). These findings suggested that accessibility of cGAS to MN with the type II NE defect was higher than that to MN with other NE assembly types, implying that cGAS accumulated more efficiently in MN with type II NE assembly than $\mathrm{MN}$ with intact and type I NE protein assemblies.

In summary, using the standard condition and two pharmacological conditions, we found three types of NE protein assembly associated with MN. Among them, the type II NE protein assembly defect was interesting because it contained a region deficient for both core and non-core NE proteins. Intriguingly, $\mathrm{MN}$ with this type of NE assembly contained unevenly distributed DNA and accumulated cGAS, a sensor of non-self-DNA, implying perturbation of not only NE but also DNA/chromatin assemblies. Because it is increasingly clear that NE assembly defects are the primary cause of genomic instability associated with MN and play a pivotal role in cell fate decision [5] [6] [7] [8] [14], our findings concerning the pharmacological settings and a method of discriminating NE proteins may be helpful for researchers in cell biology fields relevant to genome evolution, cancer development, and anti-cancer responses.

\section{Acknowledgements}

We thank all the members of the Saitoh Laboratory for helpful discussion. This work was supported by JP17H01878 and The Novartis Foundation Japan for the Promotion of Science to H.S. This work was also supported by JSPS KAKENHI Grant Numbers JP18H06069 to Y.I., JP18H05531, the research grants from The Naito Foundation and Takeda Science Foundation to N.S. We thank Dr. Toru Hirota for providing the HeLa-Kyoto cells expressing EGFP-H2B. 


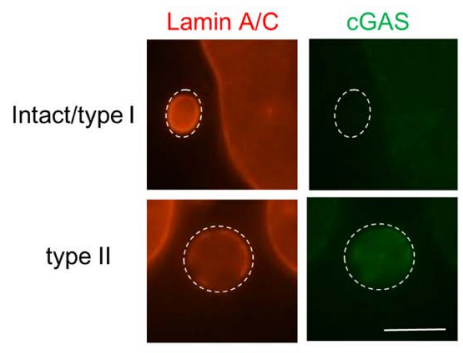

(a)

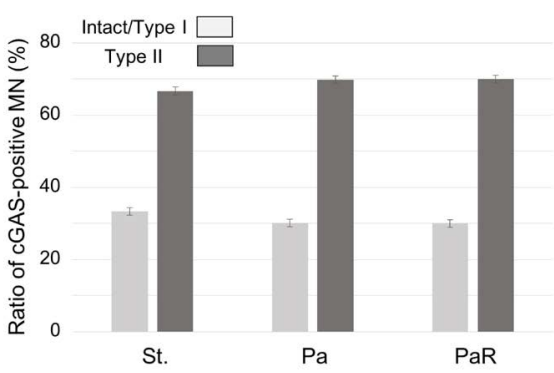

(b)

Figure 4. cGAS accumulation in MN with type II NE assembly under the standard condition and two pharmacological conditions. (a) Representative images of $\mathrm{MN}$ visualized by anti-lamin A/C (left) and anti-cGAS (right) antibodies. Lamin A/C-intact $\mathrm{MN}$ were considered as MN with "intact/type I" NE assembly (upper). Lamin A/C-deficient MN were interpreted as MN with "type II" NE assembly (lower). Scale bar: $10 \mu \mathrm{m}$; (b) Ratio of cGAS-positive MN with seemingly no defects in lamin A/C ("intact/type I" NE assembly) indicated by a light gray box. The ratio of cGAS-positive MN containing a region deficient for lamin A/C ("type II" NE assembly) is indicated by a dark gray box. Twenty MN were detected under the asynchronous culture conditions (St; left bar graph), low paclitaxel concentration conditions ( $\mathrm{Pa}$; middle bar graph), and paclitaxel plus reversine conditions (PaR; right bar graph). The bars and range indicate the mean $\pm 95 \%$ confidence interval of three independent experiments.

\section{Conflicts of Interest}

The authors declare no conflicts of interest regarding the publication of this paper.

\section{References}

[1] Ungricht, R. and Kutay, U. (2017) Mechanisms and Functions of Nuclear Envelope Remodelling. Nature Reviews Molecular Cell Biology, 18, 229-245. https://doi.org/10.1038/nrm.2016.153

[2] Dechat, A., Gajewski, A., Korbei, B., Gerlich, D., Daigle, N., Haraguchi, T., Furukawa, K., Ellenberg, J. and Foisner. R. (2004) LAP2alpha and BAF Transiently Localize to Telomeres and Specific Regions on Chromatin during Nuclear Assembly. Journal of Cell Science, 117, 6117-6128. https://doi.org/10.1242/jcs.01529

[3] Haraguchi, T., Kojidani, T., Koujin, T., Shimi, T., Osakada, H. Mori, C., Yamamoto, A. and Hiraoka, Y. (2008) Live Cell Imaging and Electron Microscopy Reveal Dynamic Processes of BAF-Directed Nuclear Envelope Assembly. Journal of Cell Science, 121, 2540-2554. https://doi.org/10.1242/jcs.033597

[4] LaJoie, D. and Ullman, K.S. (2017) Coordinated Events of Nuclear Assembly. Current Opinion in Cell Biology, 46, 39-45. https://doi.org/10.1016/j.ceb.2016.12.008 
[5] Hatch, E.H., Fischer, A.H., Deerinck, T.J. and Hetzer, M.W. (2013) Catastrophic Nuclear Envelope Collapse in Cancer Cell Micronuclei. Cell, 154, 47-60. https://doi.org/10.1016/j.cell.2013.06.007

[6] Ly, P. and Cleveland, D.W. (2017) Rebuilding Chromosomes after Catastrophe: Emerging Mechanisms of Chromothripsis. Trends in Cell Biology, 27, 917-930. https://doi.org/10.1016/j.tcb.2017.08.005

[7] Hintzsche, H., Hemmann, U., Poth, A., Utesch, D., Lott, J., Stopper, H. and Working Group “In Vitro Micronucleus Test”, Gesellschaft für Umwelt-Mutationsforschung (GUM, German-Speaking Section of the European Environmental Mutagenesis and Genomics Society EEMGS) (2017) Fate of Micronuclei and Micronucleated Cells. Mutation Research, 771, 85-98. https://doi.org/10.1016/j.mrrev.2017.02.002

[8] Liu, S., Kwon, M., Mannino, M., Yang, N., Renda, F., Khodjakov, A. and Pellman, D. (2018) Nuclear Envelope Assembly Defects Link Mitotic Errors to Chromothripsis. Nature, 561, 551-555. https://doi.org/10.1038/s41586-018-0534-Z

[9] Jordan, M.A., Wendell, K., Gardiner, S., Derry, W.B., Copp, H. and Wilson, L. (1996) Mitotic Block Induced in HeLa Cells by Low Concentrations of Paclitaxel (Taxol) Results in Abnormal Mitotic Exit and Apoptotic Cell Death. Cancer Research, 56, 816-825.

[10] Chen, J.G. and Horwitz, S.B. (2002) Differential Mitotic Responses to MicrotubuleStabilizing and -Destabilizing Drugs. Cancer Research, 62, 1935-1938.

[11] Mitchison, T.J., Pineda, J., Shi, J. and Florian, S. (2017) Is Inflammatory Micronucleation the Key to a Successful Anti-Mitotic Cancer Drug? Open Biology, 7, 170182. https://doi.org/10.1098/rsob.170182

[12] Samwer, M., Schneider, M.W.G., Hoefler, R., Schmalhorst, P.S., Jude, J.G., Zuber, J. and Gerlich. D.W. (2017) DNA Cross-Bridging Shapes a Single Nucleus from a Set of Mitotic Chromosomes. Cell, 170, 956-972. https://doi.org/10.1016/j.cell.2017.07.038

[13] Zierhut, C., Yamaguchi, N., Paredes, M., Luo, J.-D., Carrol, T. and Funabiki, H. (2019) The Cytoplasmic DNA Sensor cGAS Promotes Mitotic Cell Death. Cell, 178, 302-315. https://doi.org/10.1016/j.cell.2019.05.035

[14] Mackenzie, K.J., Carroll, P., Martin, C.A., Murina, O., Fluteau, A., Simpson, D.J., Olova, N., Sutcliffe, H., Rainger, J.K., Leitch, A., Osborn, R.T., Wheeler, A.P., Nowotny, M., Gilbert, N., Chandra, T., Reijns, M.A.M. and Jackson, A.P. (2017) cGAS Surveillance of Micronuclei Links Genome Instability to Innate Immunity. Nature, 548, 461-465. https://doi.org/10.1038/nature23449

[15] Ablasser, A. and Chen, Z.J. (2019) cGAS in Action: Expanding Roles in Immunity and Inflammation. Science, 363, eaat8657. https://doi.org/10.1126/science.aat8657

[16] Motwani, M., Pesiridis, S. and Fitzgerald, K.A. (2019) DNA Sensing by the cGAS-STING Pathway in Health and Disease. Nature Reviews Genetics, 20, 657-674. https://doi.org/10.1038/s41576-019-0151-1 


\section{Supplementary Information}

\section{1) Stock solutions}

Reagents and stock solutions used in our experiments were as follows. Chemicals were purchased from FUJIFILM Wako Pure Chemical Corp., unless otherwise stated.

a) Dulbecco's modified Eagle's medium nutrient mixture F-12 Ham at $4^{\circ} \mathrm{C}$.

b) Fetal calf serum (GE Healthcare HyClone) at $-80^{\circ} \mathrm{C}$.

c) Penicillin-streptomycin (100x) solution at $4^{\circ} \mathrm{C}$.

d) Phosphate buffered saline (PBS): $137 \mathrm{mM} \mathrm{NaCl}, 2.7 \mathrm{mM} \mathrm{KCl}, 10 \mathrm{mM}$ $\mathrm{Na}_{2} \mathrm{HPO}_{4}$, and $1.8 \mathrm{mM} \mathrm{KH}_{2} \mathrm{PO}_{4}$ at room temperature.

e) $20 \%$ triton $\times 100$ (polyoxyethylene octylphenyl ether) in PBS at $4^{\circ} \mathrm{C}$.

f) Bovine serum albumin (BSA; Takara Bio Inc.) at $4^{\circ} \mathrm{C}$.

g) $1 \mathrm{mg} / \mathrm{ml}$ reversine (Funakoshi) in DMSO at $-20^{\circ} \mathrm{C}$.

h) $1 \mathrm{mg} / \mathrm{ml}$ pactilaxel in DMSO at $-20^{\circ} \mathrm{C}$.

i) $10 \mathrm{mg} / \mathrm{ml}$ Hoechst 33342 (Dojindo) in $\mathrm{H}_{2} \mathrm{O}$ at $-20^{\circ} \mathrm{C}$.

j) Anti-fade mounting solution (Slowfade Diamond Antifade Mountant) at $-20^{\circ} \mathrm{C}$.

k) $4 \%$ Paraformaldehyde in PBS at $-20^{\circ} \mathrm{C}$.

\section{2) Antibodies}

a) Anti-RanBP2 were obtained from Dr. Nishitani, University of Hyogo (see N. Yokoyama et al. Nature 376: 184-188, 1995).

b) Anti-lamin A/C mouse monoclonal antibody from Santa Cruz Biotechnology, Inc.

c) Anti-lamin B1 rabbit polyclonal antibodies from Abcam.

d) Anti-emerin mouse monoclonal antibody from Santa Cruz Biotechnology, Inc.

e) Anti-cGAS rabbit monoclonal antibody from Cell Signaling Technology.

f) Secondary antibodies used were purchased from Jackson Immuno Research, Life Technologies, Santa Cruz Biotechnology and Medical and Biological Laboratories.

\section{3) Supplementary table}

Table S1. List of core- and non-core-NE proteins [2] [3] [8].

\begin{tabular}{cc}
\hline Core-NE proteins & Non-core-NE proteins \\
\hline LaminA & Lamin B \\
Emerin & LBR \\
BAF & SUN1 \\
LAP2 $\alpha$ & Nup133 \\
MAN1 & Nup153 \\
LEM2 & POM121 \\
SUN2 & RanBP2/Nup358
\end{tabular}




\section{4) Supplementary figures and figure legends}

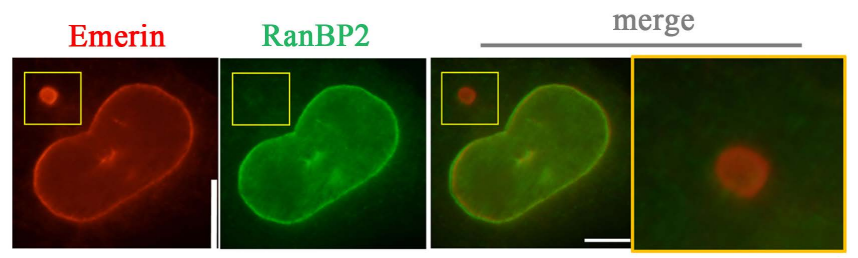

(a)

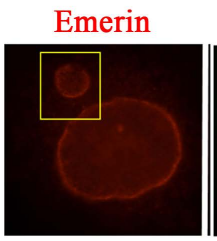

RanBP2

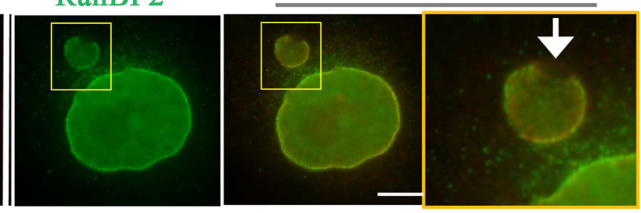

(b)

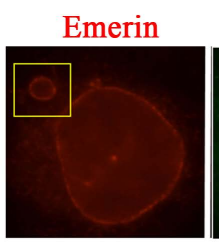

RanBP2
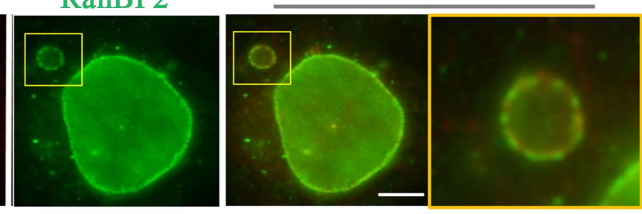

(c)

Figure S1. Association of three types of NE assembly with MN in asynchronously cultured HeLa cells. (a)-(c): Exponentially growing HeLa cells subjected to immunostaining analysis using anti-emerin (left) and anti-RanBP2 (middle-left) antibodies. Merged images are shown in the middle-right. The area in the yellow box is enlarged to the right. The arrow in B represents the region lacking both emerin and RanBP2 signals. Scale bar: $20 \mu \mathrm{m}$.
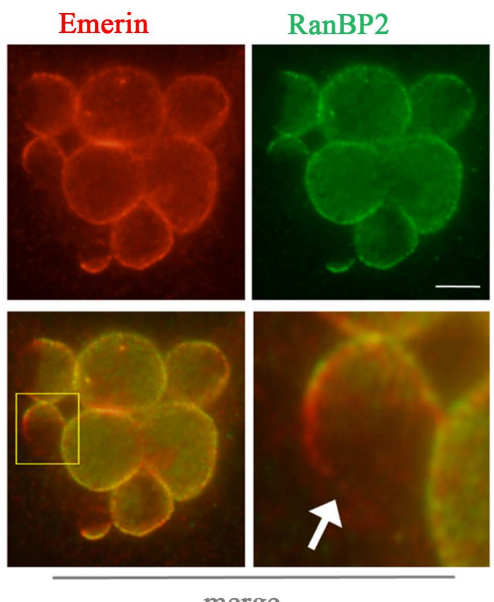

(a)
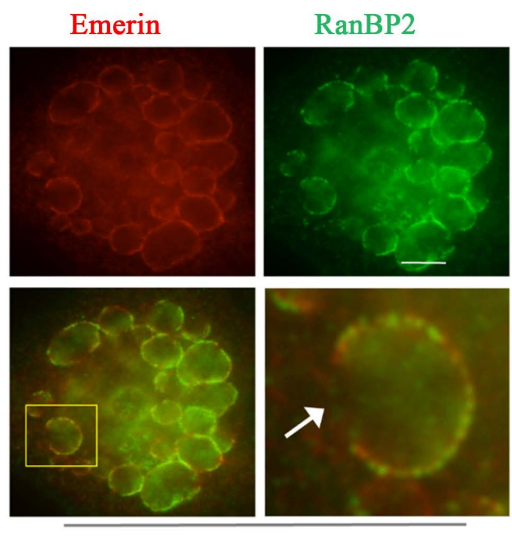

merge

(b)

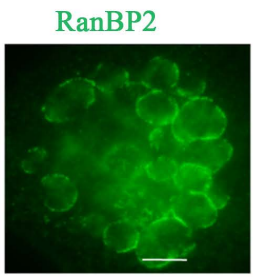

Figure S2. MN with type II NE assembly under the two pharmacological conditions. (a) MN induced under Pa conditions were visualized by anti-emerin (upper-left) and anti-RanBP2 (upper-left) antibodies. A merged image is shown at the lower-left. The yellow box is enlarged at the lower-right. The arrow represents the region lacking both emerin and RanBP2 signals. Bar: $20 \mu \mathrm{m}$; (b) MN induced under PaR conditions were visualized by anti-emerin (upper-left) and anti-RanBP2 (upper-right) antibodies. A merged image is shown at the lower-left. The yellow box is enlarged at the lower-right. The arrow represents the region lacking both emerin and RanBP2 signals. Scale bar: $20 \mu \mathrm{m}$. 

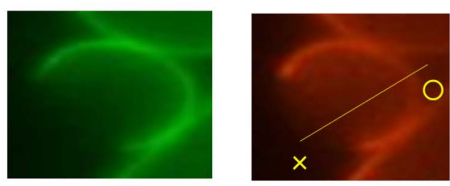

Lamin B1

Type II

$\left(\begin{array}{l}\text { Lamin A/C - } \\ \text { Lamin B1 - }\end{array}\right)$

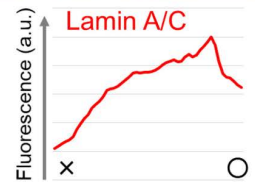

(a)
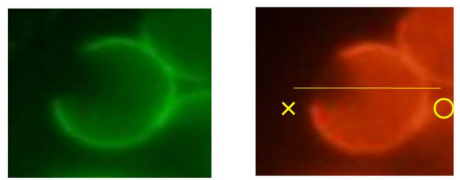

Lamin B1

Type II

$\left(\begin{array}{l}\text { Lamin A/C - } \\ \text { Lamin B1 - }\end{array}\right)$

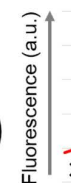

Lamin A/C

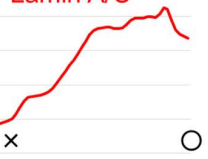

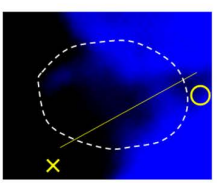

DNA

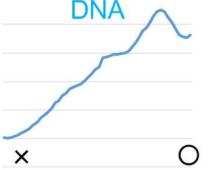

O
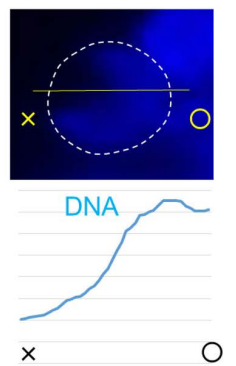

(b)

Figure S3. (a) A representative image of $\mathrm{MN}$ containing a region deficient in both lamin B1 (upper-left) and lamin A/C (upper-middle), which was detected under the Pa conditions. The DNA distribution was visualized by Hoechst 33342 staining (upper-right). Intensities of lamin A/C and Hoechst 33342 staining were measured along the indicated lines $(x \rightarrow O)$ and are represented in line graphs accordingly (lower panels). The fluorescence intensities are indicated as arbitrary units (a. u.); (b) A representative image of MN containing the region deficient in both lamin B1 (upper-left) and lamin A/C (upper-middle), which was detected under the PaR conditions, is represented. The DNA distribution was visualized by Hoechst 33342 staining (upper-right). Intensities of lamin A/C and Hoechst 33342 staining were measured along the indicated lines $(x \rightarrow O)$ and are represented in line graphs accordingly (lower panels). The fluorescence intensities are indicated as arbitrary units (a. u.).

\section{5) Supplementary procedure}

\section{Time-lapse imaging}

HeLa-Kyoto cells expressing enhanced green fluorescent protein fused to histone $\mathrm{H} 2 \mathrm{~B}$ subunit (EGFP-H2B) were maintained as described previously [Hirota et al. 2004] $]^{1}$. For image analysis of the low paclitaxel treatment, cells were seeded onto a 35-mm glass bottom dish (Matsunami) and synchronized at the G1/S boundary using a double thymidine block. Synchronized cells were released into phenol red-free Dulbecco's modified Eagle's medium/Ham's F-12 nutrient mixture containing $10 \%$ fetal calf serum and $5 \mathrm{ng} / \mathrm{ml}(\sim 6 \mathrm{nM})$ paclitaxel. For the high paclitaxel and reversine conditions, cells were treated with $1 \mu \mathrm{M}$ paclitaxel for $16 \mathrm{~h}$ to arrest them at mitosis, then $200 \mathrm{ng} / \mathrm{ml}$ reversine was added to the medium just before imaging. The dish was transferred to the cell chamber of a Keyence BZ-X810 microscope imaging system equipped with an S PlanFluor ELWD ADM 20xC objective lens. Images were captured for $\sim 24 \mathrm{~h}$ (low paclitaxel) or $\sim 2 \mathrm{~h}$ (high in I and II in Mitotic Chromosome Assembly. J. Cell Sci., 117, 6435-6445. 
paclitaxel and reversine) at every 5 min using BZ-X800 Analyzer (Keyence).

6) Supplementary movie legends

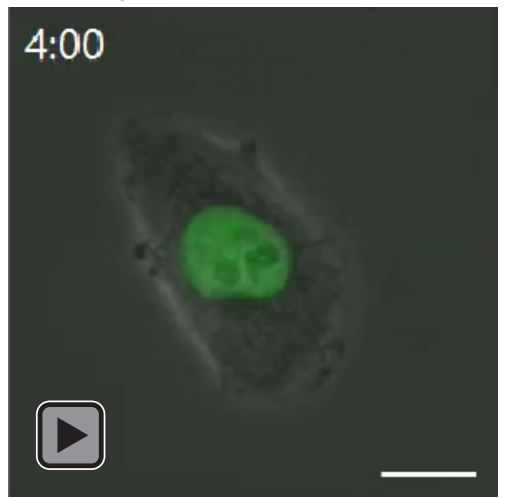

Movie S1. Time-lapse observation of GFP-H2B-expressing HeLa cells exposed to low concentration paclitaxel, leading to MN formation. Time-lapse images were obtained under a green fluorescence field at lower magnification $(\times 20)$ and reconstructed as a movie. Scale bar: $20 \mu \mathrm{m}$.

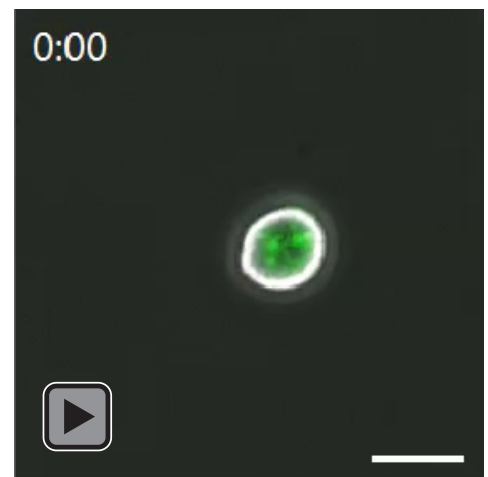

Movie S2. Time-lapse observation of GFP-H2B-expressing HeLa cells treated with high concentration paclitaxel, followed by reversine-exposure. Time-lapse images were obtained under a green fluorescence field at lower magnification $(\times 20)$ and reconstructed as a movie. Scale bar: $20 \mu \mathrm{m}$.

\section{7) Graphic abstract}

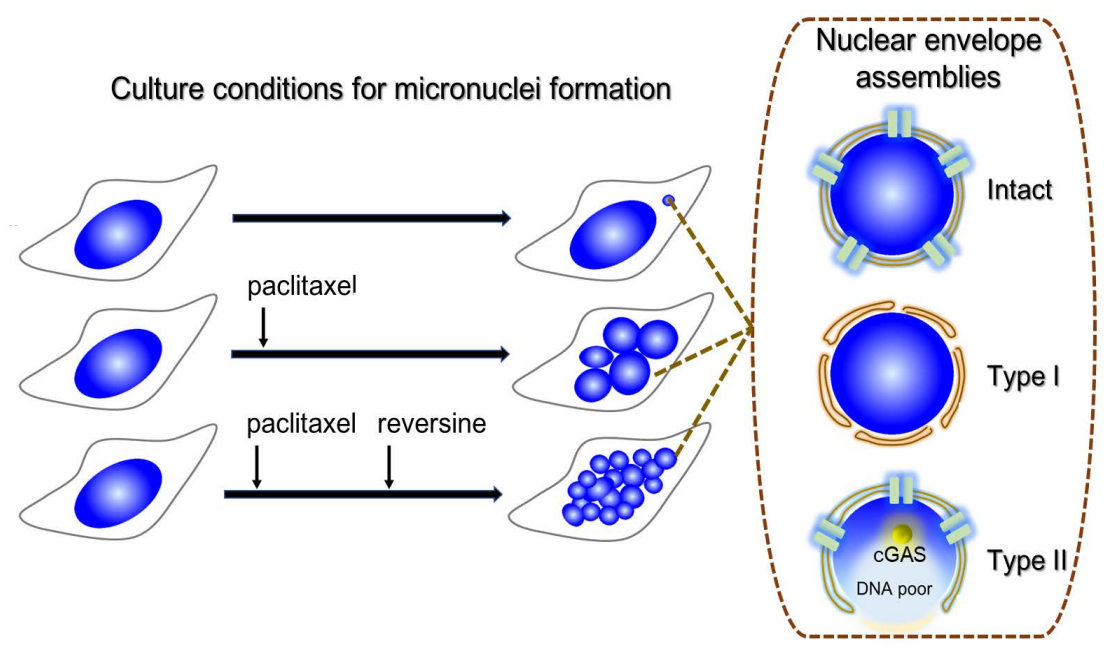




\section{8) Highlights}

a) Micronucleus (MN) formation was assessed under three cell culture conditions.

b) Three types of nuclear envelope (NE) assemblies associated with MN were detected.

c) Type II NEs contain a region deficient for core and non-core NE proteins.

d) A DNA-poor and cGAS-enriched area is present in MN with type II NE assembly.

e) Type II NE assembly may be linked to cell fate decision of MN-containing cells.

\section{Abbreviations}

cGAS: cyclic Guanosine monophosphate-Adenosine monophosphate (GMP-AMP) Synthase

MN: Micronucleus/Micronuclei

NE: Nuclear Envelope

Pa: Paclitaxel

PBS: Phosphate Buffered Saline

R: Reversine 